\title{
Molecular Dynamics Simulation of Mechanical Properties for $\alpha$-SiO 2 Crystal
}

\author{
Jierong Guo, Lei Ma \\ College of Mathematics and Physics Science, Hunan University of Arts and Science, Changde, China \\ Email: jierong_guo@126.com, mlm16277@126.com
}

How to cite this paper: Guo, J.R. and Ma, L. (2019) Molecular Dynamics Simulation of Mechanical Properties for $\alpha-\mathrm{SiO}_{2}$ Crystal. Journal of Modern Physics, 10, 577-584. https://doi.org/10.4236/jmp.2019.106040

Received: April 1, 2019

Accepted: April 23, 2019

Published: April 26, 2019

Copyright $\odot 2019$ by author(s) and Scientific Research Publishing Inc. This work is licensed under the Creative Commons Attribution International License (CC BY 4.0).

http://creativecommons.org/licenses/by/4.0/

\section{cc) (i) Open Access}

\begin{abstract}
The mechanical properties of the $\alpha-\mathrm{SiO}_{2}$ crystal are studied by molecular dynamics method with Tersoff potential function. The results show that the $\alpha-\mathrm{SiO}_{2}$ crystal goes through elastic deformation, plastic deformation and fracture deformation in the process of uniaxial loading at room temperature. The $\alpha-\mathrm{SiO}_{2}$ is from crystal phase transformation to amorphous phase in plastic deformation. And also by studying the influence of temperature on the tensile mechanical properties of $\alpha-\mathrm{SiO}_{2}$, it finds that the yield strength and elastic modulus of $\alpha-\mathrm{SiO}_{2}$ decrease gradually as the temperature increases. Moreover, the higher the temperature, the lower the fracture stress and fracture strain; the $\alpha-\mathrm{SiO}_{2}$ crystal is easy to be broke under high temperature uniaxial loading. And it also finds that the crack is able to decrease the mechanical properties of $\alpha-\mathrm{SiO}_{2}$ crystal.
\end{abstract}

\section{Keywords}

Mechanical Properties, Tensile, Molecular Dynamics

\section{Introduction}

The $\alpha-\mathrm{SiO}_{2}$ is the stable crystal at normal temperature. The research for $\alpha-\mathrm{SiO}_{2}$ crystal is mainly focused on the phase transition of amortization under high pressure. For example, Wang et al. [1] study the structural transformation of $\alpha-\mathrm{SiO}_{2}$ crystal under high temperature and high pressure, and point out that $\alpha-\mathrm{SiO}_{2}$ crystal is able to be synthesized on a small scale coesite under high temperature and high pressure. Palmer et al. [2] find the phase transition rule of $\alpha-\mathrm{SiO}_{2}$ by studying the pressurized phase transition process at room temperature. Zhang et al. [3] study the structural transformation of $\alpha-\mathrm{SiO}_{2}$ crystal at high temperature and high pressure and the experimental conditions of coesite formation through mechanical ball milling. Dubrovinsky et al. [4] study the 
high-pressure phase transition of $\alpha-\mathrm{SiO}_{2}$, the results show that it obtains another phase structure of $\alpha-\mathrm{SiO}_{2}$ similar to the structure of $\alpha-\mathrm{PbO}_{2}$, and find that the stress between $25 \mathrm{GPa}$ and $35 \mathrm{GPa}$ resulting in an amorphous transformation, which is completely amorphous after unloading stress. However, with the rapid development of computer in recent years, it has become possible to study the mechanical properties, structural transformation and physical characteristics of crystal materials by means of large-scale parallel computation using molecular dynamics simulation [5] [6] [7] [8] [9]. For instance, Pan [10] simulates the high-pressure phase transition of $\alpha-\mathrm{SiO}_{2}$ with Morse potential using molecular dynamics simulation, and points out that the amorphous phase transition occurs when the stress of crystal is higher than $24.6 \mathrm{GPa}$, and the calculated results are in agreement with the experimental results. Therefore, computer simulations open up another effective way to study the mechanical properties of crystal, which can provide theoretical support for experimental research.

This paper is focused on investigating the basic mechanical properties of $\alpha-\mathrm{SiO}_{2}$ using molecular dynamics simulation. The stress-strain curves of $\alpha-\mathrm{SiO}_{2}$ have been studied with the temperature increasing. And the variation of yield stress, yield strain and elastic modulus is discussed for $\alpha-\mathrm{SiO}_{2}$ crystal in the process of uniaxial tension. The influence law of temperature on tensile mechanical properties is also discussed to comprehensively evaluate the mechanical properties of $\alpha-\mathrm{SiO}_{2}$. Meanwhile, it constructs the crack models of $\alpha-\mathrm{SiO}_{2}$ and analyzes the influence of crack on mechanical properties.

\section{Atomistic Model and Simulation Method}

Figure 1 shows the crystal structure of $\alpha-\mathrm{SiO}_{2}$. In silicon dioxide, silicon is located at the center of the tetrahedron, and four oxygen atoms are located at the four top angles of the tetrahedron. And then put the tetrahedron structure of $\mathrm{SiO}_{2}$ into the body-center cubic and construct a crystal cell of $\alpha-\mathrm{SiO}_{2}$ as shown in Figure 1(A). Then the crystal structure of $\alpha-\mathrm{SiO}_{2}$ is established according to the Cartesian coordinates as shown in Figure 1(B). Where, the lattice constant $a=$ 4.978 angstrom, $b=4.978$ angstrom, $c=6.948$ angstrom, the length of the box is $30 a \times 30 b \times 30 c$, the total number of atoms is 324,000 , and periodic boundary conditions are used in $x^{-}, y$ - and $z$-directions.

In molecular dynamics (MD) simulation, the Tersoff potential function [11] is adopted to describe the interaction between $\mathrm{Si}$ and $\mathrm{O}$. the model is equilibrated under NPT ensemble at $300 \mathrm{k}$, and a Nose-Hoover thermostat is applied to maintain the system temperature. Figure 2 present the energy variation of the model in relaxation process, it can be obtained that the crystal model reaches the equilibrium after $10 \mathrm{ps}$. Then, the model is performed to uniaxial tensile test in $z$-direction using MD simulation with the time integration step of $1 \mathrm{fs}$ under NPT ensemble. At the same time, the temperatures of $300 \mathrm{~K}, 500 \mathrm{~K}, 700 \mathrm{~K}$ and $900 \mathrm{~K}$ are selected to simulate the tensile mechanical properties of $\alpha$-SiO ${ }_{2}$ crystals at different temperatures. And the LAMMPS code [12] is applied to simulate the tensile mechanical properties. 


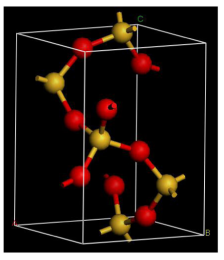

(A)

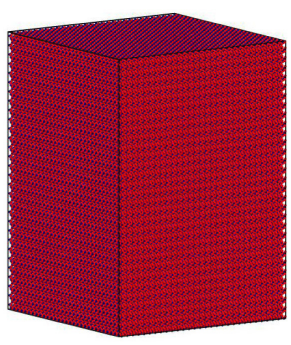

(B)

Figure 1. The initial model of $\alpha-\mathrm{SiO}_{2}$.

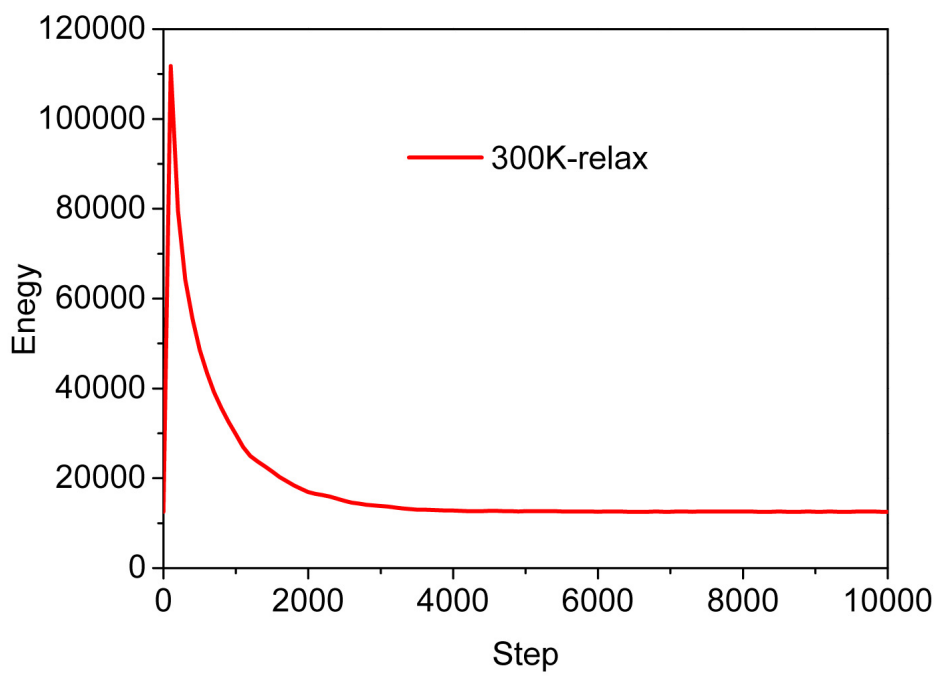

Figure 2. The energy variation of $\alpha-\mathrm{SiO}_{2}$ in relaxation process.

\section{Results and Discussion}

\subsection{Analysis of Stress-Strain Curve}

Figure 3 shows the stress-strain curve of $\alpha-\mathrm{SiO}_{2}$ under uniaxial tension at room temperature. The yield strain, yield stress, fracture strength and deformation characteristic and so on can be obtained by stress-strain curve, which can reflect the basic mechanical properties of the material. It can be seen from the Figure 3 that the elastic limit of $\alpha-\mathrm{SiO}_{2}$ crystal appears when the strain is up to $4.7 \%$ (as shown in Figure 3(A)), and the stress-strain curve present non-linear relationship. After $\mathrm{A}$, the $\alpha-\mathrm{SiO}_{2}$ crystal enters the elastic deformation stage. When the strain is up to $32.6 \%$, the stress reaches the first yield point, the yield stress is $22.6 \mathrm{GPa}$. Then the $\alpha-\mathrm{SiO}_{2}$ crystal enters the plastic deformation stage, the stress oscillates between point $\mathrm{B}$ and point $\mathrm{C}$ with the strain increasing, this moment the nanocrystal appears amorphous phase transition. When the stress passes $C$ in Figure 3, the crystal structure is completely amorphous, the stress reaches the peak value-fracture strength ( $36 \mathrm{GPa}$ ), the $\alpha-\mathrm{SiO}_{2}$ crystal enter the stage of fracture. Then the stress falls rapidly to zero as the strain increases and the crystal breaks completely. It can be seen from the Figure 3 that $\alpha-\mathrm{SiO}_{2}$ crystal experiences the elastic stage, the plastic stage (amorphous phase transition) and the 


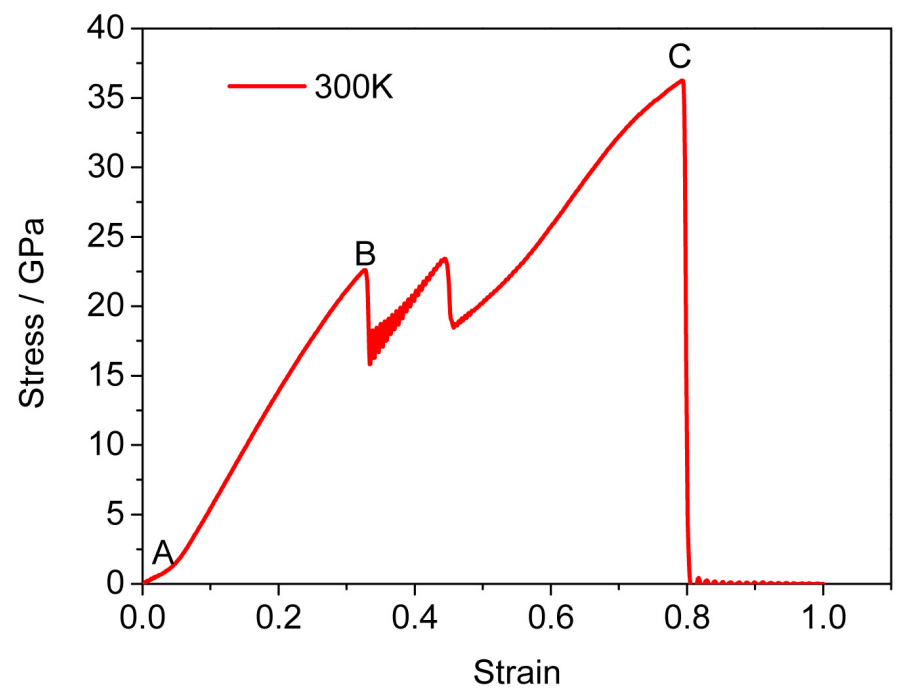

Figure 3. The stress-strain curve of $\alpha-\mathrm{SiO}_{2}$ in uniaxial tension at room temperature.

fracture stage during the uniaxial tensile process at room temperature, and the elastic modulus reached $69 \mathrm{GPa}$, so the $\alpha-\mathrm{SiO}_{2}$ crystal has excellent strength.

\subsection{Temperature Effect}

In order to discuss the influence of temperature on the tensile mechanical properties of the $\alpha-\mathrm{SiO}_{2}$ crystal, on the basis of room temperature, the temperatures of $500 \mathrm{~K}, 700 \mathrm{~K}$ and $900 \mathrm{~K}$ are selected, and then the mechanical properties of $\alpha-\mathrm{SiO}_{2}$ crystals are simulated at different temperatures. Figure 4 shows the variation of the stress-strain curves of the $\alpha-\mathrm{SiO}_{2}$ crystal at different temperatures. The simulation results show that the yield stress and yield strain decrease with the increasing of temperature. It indicated that the higher the temperature is, the shorter the elastic deformation stage of $\alpha-\mathrm{SiO}_{2}$ crystal is, and the loading strain of achieving yield strength is smaller. Meanwhile, Table 1 shows the mechanical parameters of $\alpha-\mathrm{SiO}_{2}$ under uniaxial tensile at different temperatures, the results present that the elastic modulus decreases gradually with the increasing of temperature. From the data of the calculated elastic model in Table 1, the elastic modulus of $\alpha-\mathrm{SiO}_{2}$ crystal is reduced by $2.3 \mathrm{GPa}$ when the temperature increase from $300 \mathrm{~K}$ to $500 \mathrm{~K}$. As the temperature rise from $500 \mathrm{~K}$ to $700 \mathrm{~K}$, the elastic modulus of $\alpha-\mathrm{SiO}_{2}$ crystal is reduced by $1.34 \mathrm{GPa}$. The elastic modulus decreases by $1.26 \mathrm{GPa}$ as the modulus increases from $700 \mathrm{~K}$ to $900 \mathrm{~K}$. It can be concluded that although the elastic modulus of $\alpha-\mathrm{SiO}_{2}$ crystal decreases as a result of high temperature, but there is no large range of decrease. Therefore, the high temperature only slightly reduces the strength of the $\alpha-\mathrm{SiO}_{2}$ crystal and does not have much influence on the overall strength.

However, the temperature has a great influence on the fracture strength of the $\alpha-\mathrm{SiO}_{2}$ crystal. Figure 5 shows the variation curves of fracture stress and fracture strain at different temperatures. As can be seen from the Figure 5, the fracture stress decreases from $36 \mathrm{GPa}$ to $29 \mathrm{GPa}$ with the increasing of temperature, 
Table 1. Mechanical parameters of uniaxial tensile of $\alpha-\mathrm{SiO}_{2}$ at different temperatures.

\begin{tabular}{cccc}
\hline$\alpha-\mathrm{SiO}_{2}$ & $\varepsilon_{\text {yield }}(\mathrm{GPa})$ & $\sigma_{\text {yield }}(\mathrm{GPa})$ & $E(\mathrm{GPa})$ \\
\hline $300 \mathrm{~K}$ & 0.326 & 22.6 & 69.32 \\
$500 \mathrm{~K}$ & 0.287 & 19.24 & 67.03 \\
$700 \mathrm{~K}$ & 0.274 & 18.00 & 65.69 \\
$900 \mathrm{~K}$ & 0.223 & 14.37 & 64.43 \\
\hline
\end{tabular}

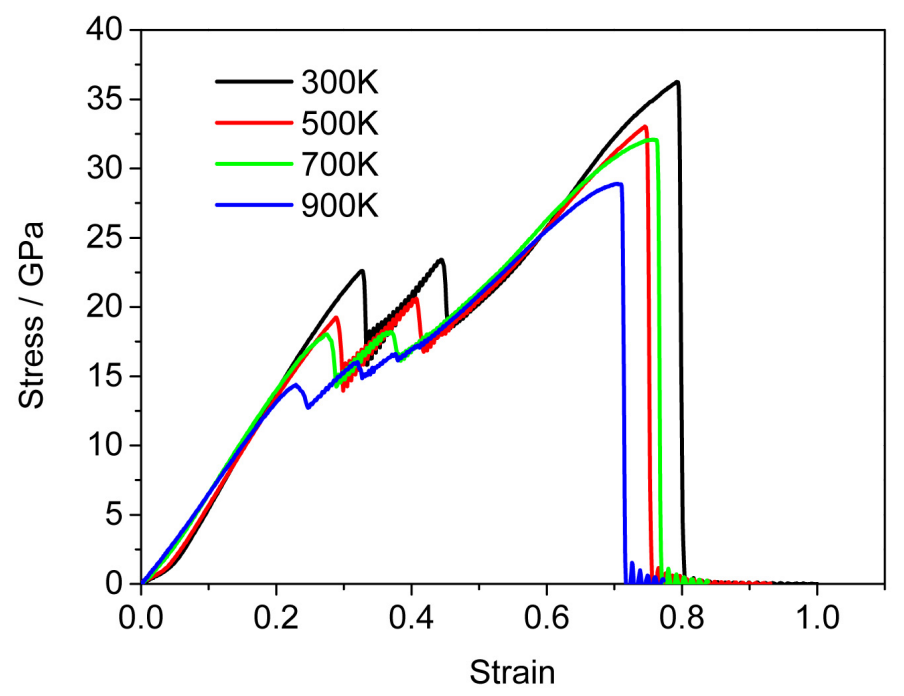

Figure 4. The tensile stress-strain curve of $\alpha-\mathrm{SiO}_{2}$ at different temperatures.

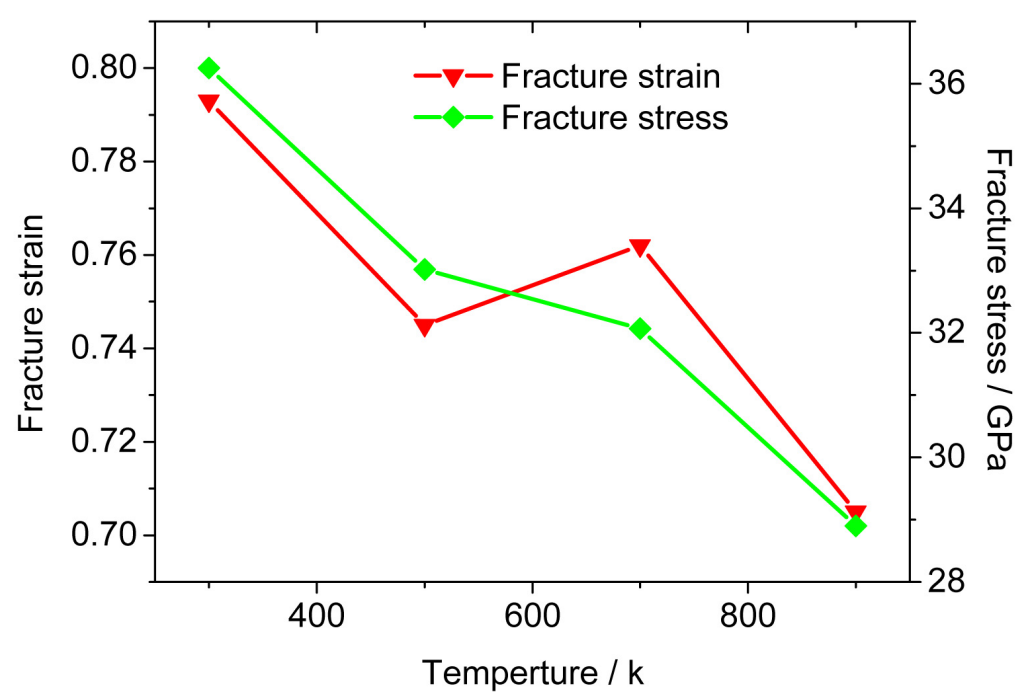

Figure 5. The fracture stress and fracture strain for $\alpha-\mathrm{SiO}_{2}$ at different temperatures.

which shows a straight downward trend. Although the fracture strain rebound in the process of decreasing with the increasing of temperature, the overall state is also in decline. Therefore, it can be concluded that the higher the temperature is, the lower the fracture stress and fracture strain are, and it is the more easily fracture for $\alpha-\mathrm{SiO}_{2}$ crystal under high temperature uniaxial loading. 


\subsection{The Mechanical Properties of Crack Propagation in $\alpha-\mathrm{SiO}_{2}$ Crystal}

In order to discuss the influence of crack on mechanical properties of $\alpha-\mathrm{SiO}_{2}$ crystal, we construct a crack model by deleting some atoms in $\alpha-\mathrm{SiO}_{2}$ crystal as shown in Figure 6, and the initial crack length is $2.98 \mathrm{~nm}$. This crack model is then uniaxial stretched for studying the effect of crack on mechanical properties. Figure 7 shows the stress-strain curve of $\alpha$-SiO ${ }_{2}$ crack model at different temperatures. The results present that the yield stress and yield strain decrease with the temperature increasing as shown in Figure 7, the reason is that the crack propagation rate increase with the temperature increasing so that the internal structure is seriously damage at high temperature. Compared to the perfect crystal of $\alpha-\mathrm{SiO}_{2}$, as the temperature increase from $300 \mathrm{~K}$ to $700 \mathrm{~K}$, the crack leads to decreasing of yield stress and yield strain. But the crack model has relatively larger yield stress and yield strain at $900 \mathrm{~K}$, which indicate that the high temperature cause the structure softening so that the crack appear collapse and inhibit the crack rapid propagation.

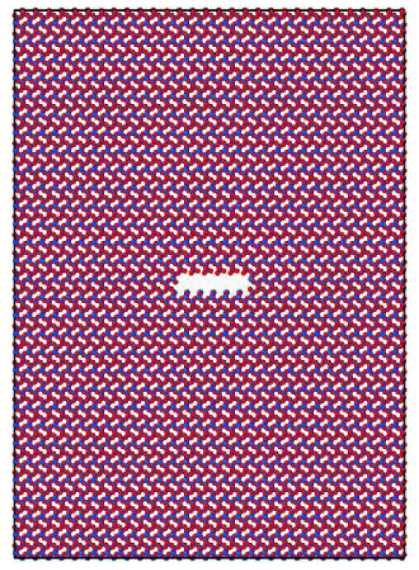

Figure 6. The crack model of $\alpha-\mathrm{SiO}_{2}$.

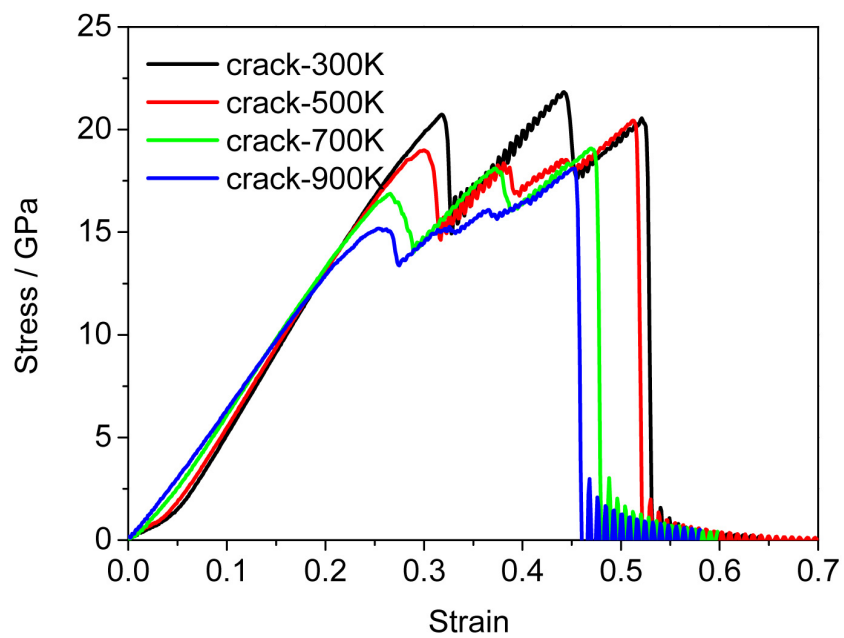

Figure 7. The tensile stress-strain curve of $\alpha-\mathrm{SiO}_{2}$ crack model at different temperatures. 
Table 2. Mechanical parameters of crack model of $\alpha-\mathrm{SiO}_{2}$ at different temperatures.

\begin{tabular}{cccc}
\hline$\alpha-\mathrm{SiO}_{2}$ & $\varepsilon_{\text {yield }}(\mathrm{GPa})$ & $\sigma_{\text {yield }}(\mathrm{GPa})$ & $E(\mathrm{GPa})$ \\
\hline $300 \mathrm{~K}$ & 0.31 & 20.7 & 66.77 \\
$500 \mathrm{~K}$ & 0.30 & 18.9 & 62.33 \\
$700 \mathrm{~K}$ & 0.26 & 16.8 & 64.61 \\
$900 \mathrm{~K}$ & 0.26 & 15.1 & 58.07 \\
\hline
\end{tabular}

And the elastic modulus is obtained by fitting the stress-strain curve of the $\alpha$ - $\mathrm{SiO}_{2}$ crack model. The calculated values show in Table 2, which present that the elastic modulus of $\alpha$ - $\mathrm{SiO}_{2}$ crack model vary from $66 \mathrm{GPa}$ to $58 \mathrm{GPa}$ as the temperature arising. Compared to the perfect crystal of $\alpha-\mathrm{SiO}_{2}$, the elastic modulus of crack model is decreasing at each temperature. So the crack causes the mechanical properties to drop.

\section{Conclusion}

The tensile mechanical properties of $\alpha-\mathrm{SiO}_{2}$ crystal are simulated by means of molecular dynamics and with Tersoff potential function. The results show that the $\alpha-\mathrm{SiO}_{2}$ crystal goes through elastic deformation, plastic deformation and fracture deformation in the process of uniaxial loading at room temperature, and the yield strength is $22.6 \mathrm{GPa}$, the fracture strength is $36 \mathrm{GPa}$. The $\alpha-\mathrm{SiO}_{2}$ is from crystal phase transformation to amorphous phase transition in plastic deformation. And also by studying the influence of temperature on the tensile mechanical properties of $\alpha$ - $\mathrm{SiO}_{2}$, it finds that the yield strength and elastic modulus of $\alpha-\mathrm{SiO}_{2}$ decrease gradually as the temperature increases. Moreover, the higher the temperature, the lower the fracture stress and fracture strain; the $\alpha-\mathrm{SiO}_{2}$ crystal is easy to be broke under high temperature uniaxial loading. The yield stress and yield strain decrease with the temperature increases. For the crack model of $\alpha-\mathrm{SiO}_{2}$, the yield stress and yield strain decrease with the temperature increases comparing to the perfect crystal of $\alpha-\mathrm{SiO}_{2}$, and the crack causes the mechanical properties to drop.

\section{Acknowledgements}

This work is supported by Hunan natural science foundation (No. 2016JJ5002) and the Doctor Start project of Hunan University of Arts and Science (No. 16BSQD05).

\section{Conflicts of Interest}

The authors declare no conflicts of interest regarding the publication of this paper.

\section{References}

[1] Wang D.J. (2010) A Study on the Structure Transition of $\alpha$-Quartz under High 
Temperature and High Present. Journal of Changchun University, 20, 14-16.

[2] Palmer, D.C., Hemley, R.J. and Prewitt, C.T. (1994) Raman Spectroscopic Study of High-Pressure Phase Transitions in Cristobalite. Physics and Chemistry of Minerals, 21, 481-488. https://doi.org/10.1007/BF00203922

[3] Zhang, G.Q., Xe, D.P., Wang, D.O., et al. (2008) Structural Transition of Nanometer $\mathrm{SiO} 2$ Powders under High Pressure and High Temperature. Journal of Jilin University, 46, 311-313.

[4] Dubrovinsky, L.S., Dubrovinskaia, N.A., Saxena, S.K., et al. (2001) Pressure-Induced Transitions of Cristobalite. Chemical Physics Letter, 333, 264-270. https://doi.org/10.1016/S0009-2614(00)01147-7

[5] Wang, K., Xiao, S.F., Deng, H.Q., et al. (2014) An Atomic Study on the Shock-Induced Plasticity and Phase Transition for Iron-Based Single Crystals. International Journal of Plasticity, 59, 180-198. https://doi.org/10.1016/j.ijplas.2014.03.007

[6] Ma, L., Xiao, S.F., Deng, H.Q. and Hu, W.Y. (2014) Molecular Dynamics Simulation of Fatigue Crack Propagation in Bcc Iron under Cyclic Loading. International Journal of Fatigue, 68, 253. https://doi.org/10.1016/j.ijfatigue.2014.04.010

[7] Ma, L., Xiao, S.F., Deng, H.Q. and Hu, W.Y. (2015) Atomic Simulation of Fatigue Crack Propagation in Ni3Al. Applied Physics A, 118, 1399-1406. https://doi.org/10.1007/s00339-014-8895-0

[8] Ma, L., Xiao, S.F., Deng, H.Q. and Hu, W.Y. (2016) Tensile Mechanical Properties of Ni-Based Superalloy of Nanophases Using Molecular Dynamic Simulation. Physica Status Solid, 253, 716-732. https://doi.org/10.1002/pssb.201552149

[9] Ma, L., Zhong, Y. and Wen, X.X. (2011) Process in Nanocrystalline Metal under Tensile Stress. Materials Review, 25, 10-13.

[10] Pan, H.B. (200) A New Phase Transition of Quartz- $\alpha$ SiO2 by Molecular by Dynamics Simulation. Acta Mineralogica Sinica, 21, 511-514.

[11] Teodoro Laino, M., et al. (2007) Interatomic Potential for Si-O Systems Using Tersoff Parameterization. Computational Materials Science, 39, 334-339. https://doi.org/10.1016/j.commatsci.2006.06.010

[12] http://lammps.sandia.gov 